\title{
High order Riesz transforms and mean value formula for generalized translate operator
}

\author{
Ismail Ekincioglu ${ }^{1 *}$, Hasan H Sayan² and Cansu Keskin ${ }^{1}$
}

${ }^{\text {*Correspondence: }}$

ismail.ekincioglu@dpu.edu.tr

'Department of Mathematics,

Dumlupınar University, Kütahya,

Turkey

Full list of author information is

available at the end of the article

\begin{abstract}
In this paper, the mean value formula depends on the Bessel-generalized shift operator corresponding to the solutions of the boundary value problem related to the multidimensional Bessel operator are studied. In addition, Riesz transforms $R_{\mathrm{B}}$ related to the multidimensional Bessel operators are studied. Since a Bessel-generalized shift operator is a translation operator corresponding to the multidimensional Bessel operator, we construct a family of $R_{\mathrm{B}}$ by using a Bessel-generalized shift operator. Finally, we analyze weighted inequalities involving $R_{\mathrm{B}}$.

MSC: Primary 31B05; 35G10; secondary 34B30; 47F05
\end{abstract}

Keywords: Bessel equation; generalized shift operator; mean value theorem

\section{Introduction}

Singular integral operators are playing an important role in harmonic analysis, the theory of functions and partial differential equations. Singular integrals are associated with the $\Delta_{\mathrm{B}_{n}}$ Laplace-Bessel differential operator, which is known as an important operator in analysis and its applications; these have been the research areas of many mathematicians such as Muckenhoupt and Stein [1, 2], Kipriyanov and Klyuchantsev [3, 4], Aliev and Gadjiev [5], Guliev [6], Gadjiev. Also, singular integral operators related to the generalized shift operator were studied by [7] and [8-10] and others.

The Bessel-generalized shift operator is one of the most important generalized shift operator on the half line $\mathbb{R}_{+},[11,12]$. The Bessel-generalized translation is used, while studying various problems connected with Bessel operators [13]. Fourier-Bessel harmonic analysis, i.e. the part of harmonic analysis addressing various problems on Bessel (Hankel) integral transforms, is closely connected with Bessel-generalized shift operator.

It is well known that the fundamental solutions of the classical stationary of mathematical physics (the harmonic equation, polyharmonic equation, and Helmholtz equation) are radial functions. Therefore, it is natural to seek these solutions as solutions of ordinary differential equations. However, since the spherical coordinate transformation transforms an equation with the Laplace operator in $\mathbb{R}^{n}$ into an ordinary differential equation with the singular Bessel differential operator, interest arose (probably, a long time ago) in studying methods for constructing the fundamental solutions of singular ordinary differential equations with the Bessel operator in place of the second derivative. In this connection, it might be very useful to prove a theorem on the fundamental solution of an ordinary differential equation involving the Bessel operator with constant coefficients, similar to the well-known theorem on the fundamental solution of an ordinary differential equation.

C2014 Ekincioglu et al.; licensee Springer. This is an Open Access article distributed under the terms of the Creative Commons Attribution License (http://creativecommons.org/licenses/by/2.0), which permits unrestricted use, distribution, and reproduction in any medium, provided the original work is properly cited. 
The result obtained on the fundamental solution of an ordinary differential operator with the Bessel operator has allowed us to analyze equations with the singular differential operator $\triangle_{\mathrm{B}_{\gamma}}=\sum_{i=1}^{n} \mathrm{~B}_{\gamma_{i}}+\sum_{i=n+1}^{N} \frac{\partial^{2}}{\partial x_{i}^{2}}$ where the different indices $\gamma_{i}$ act with respect to part of the variables and these indices may take negative values. The latter fact is essential because the tools used in problems of this kind (the Poisson operator and generalized shift operator of integral nature) are defined only for $\gamma_{i}>0$. To find the fundamental solution with a singularity at an arbitrary point, we use the generalized shift operator that acts with respect to the radial variable. Note also that the mixed-type generalized shift operator, which is conventionally used in such problems, coincides with the radial shift operator on radial functions provided that $\gamma_{i}>0$. The fundamental solutions (of the $B$-harmonic and $B$-polyharmonic equations and of the singular Helmholtz equation) found coincide with the known solutions when $\gamma_{i}>0$ and with the classical solutions when $\gamma_{i}=0$.

It is well known that harmonic functions satisfy various mean value theorems, which may be considered as generalizations of the Gauss mean value theorem. There have been a number of studies on mean value theorems. Cheng [14] obtained a converse for a different mean value expression. Nicolesco [15] gave an expression in terms of certain iterated means and showed that a converse was also true. The mean value theorems for harmonic functions have also been studied by Pizetti [16], Picone [17], Ekincioglu [8], and Kipriyanova [18] and [19].

The solutions of the boundary value problems for Laplace operator are related to the ordinary shift operator. Also, the solutions of boundary value problems for Laplace-Bessel and Bessel operators are corresponding to the generalized shift operator and Besselgeneralized shift operator, respectively.

In this paper, singular integral operators generated by a Bessel-generalized shift operator are studied. In addition, the mean value formulas related to the Bessel-generalized shift operator are given.

Riesz-Bessel singular integral operators related to generalized shift operator for LaplaceBessel operator were showed in [7] and [9]. The authors used the mean value theorem related to the generalized shift operator. The mean value formula for the equations $\Delta_{\mathrm{B}_{x_{n}}} u=0$ and $\Delta_{\mathrm{B}_{x_{n}}} u+\lambda u=0$ were obtained by [8] and [19], respectively, where $\Delta_{\mathrm{B}_{x_{n}}}=\sum_{i=1}^{n} \frac{\partial^{2}}{\partial x_{i}{ }^{2}}+\frac{2 \gamma_{n}}{x_{n}} \frac{\partial}{\partial x_{n}}$.

In this study, we introduce the mean value formula for the equation $\mathrm{B} u=0$ and the high order Riesz-Bessel transform associated with the Bessel-generalized shift operator for the B Bessel differential operator

$$
\mathrm{B}=\sum_{i=1}^{n} \mathrm{~B}_{i}, \quad \mathrm{~B}_{i}=\frac{\partial^{2}}{\partial x_{i}{ }^{2}}+\frac{2 \gamma_{i}}{x_{i}} \frac{\partial}{\partial x_{i}}, \gamma_{1}>0, \ldots, \gamma_{n}>0 .
$$

Let $\mathbb{R}^{n}$ be $n$-dimensional Euclidean space and $x=\left(x_{1}, \ldots, x_{n}\right), \xi=\left(\xi_{1}, \ldots, \xi_{n}\right)$ be vectors in $\mathbb{R}^{n}$, then $x \cdot \xi=x_{1} \xi_{1}+\cdots+x_{n} \xi_{n},|x|=(x \cdot x)^{1 / 2}$. Denote $\mathbb{R}_{+}^{n}=\left\{x \in \mathbb{R}^{n}: x_{1}>0, \ldots, x_{n}>0\right\}$, $S_{+}^{n}=\left\{x \in \mathbb{R}_{+}^{n}:|x|=1\right\}, \gamma=\left(\gamma_{1}, \ldots, \gamma_{n}\right), \gamma_{1}>0, \ldots, \gamma_{n}>0,|\gamma|=\gamma_{1}+\cdots+\gamma_{n}$ and $d \mu_{\gamma}(x)=$ $\prod_{i=1}^{n} x_{i}^{2 \gamma_{i}} d x$. We shall denote by $L_{p, \gamma}\left(\mathbb{R}^{n}, d \mu_{\gamma}(x)\right)$-spaces (the Lebesgue space with respect to the measure $\mu_{\gamma}$ ), the set of all measurable functions $f$ on $\mathbb{R}_{+}^{n}$ such that the norm

$$
\|f\|_{L_{p, \gamma}} \equiv\|f\|_{p, \gamma}=\left(\int_{\mathbb{R}_{+}^{n}}|f(x)|^{p} d \mu_{\gamma}(x)\right)^{1 / p}, \quad 1 \leq p<\infty
$$


is finite. We determine the Bessel-generalized translation $T^{y} \varphi(x)=u(x), x, y \in \mathbb{R}_{+}^{n}$ of a function $\varphi(x) \in \mathcal{C}^{(2)}\left(\mathbb{R}_{+}^{n}\right)$ as the solution to the following initial value problem:

$$
\left.\begin{array}{l}
\mathrm{B}_{x} u(x, y)=\mathrm{B}_{y} u(x, y), \\
u(x, 0)=\varphi(x), \quad u_{y}(x, 0)=0
\end{array}\right\}
$$

where $B$ is the multidimensional Bessel differential operator [20]. The solution of the initial value problem (1.1) exists, is unique, and can be written explicitly as

$$
\begin{aligned}
T^{y} \varphi(x):= & c_{\gamma} \int_{0}^{\pi} \ldots \int_{0}^{\pi} \varphi\left(\left(x_{1}, y_{1}\right)_{\alpha_{1}}, \ldots,\left(x_{n}, y_{n}\right)_{\alpha_{n}}\right) \\
& \times\left(\prod_{i=1}^{n} \sin ^{2 \gamma_{i}-1} \alpha_{i}\right) d \alpha_{1} \cdots d \alpha_{n}
\end{aligned}
$$

where $c_{\gamma}=\prod_{i=1}^{n} \Gamma\left(\gamma_{i}+\frac{1}{2}\right)\left[\Gamma\left(\frac{1}{2}\right) \Gamma\left(\gamma_{i}\right)\right]^{-1}$ and $\left(x_{i}, y_{i}\right)_{\alpha_{i}}=\sqrt{x_{i}^{2}+y_{i}^{2}-2 x_{i} y_{i} \cos \alpha_{i}}, 1 \leq i \leq n$. By (1.2), the operator $T^{y}$ can be extended to all functions $L_{p, \gamma}\left(\mathbb{R}_{+}^{n}\right)$. The operator $T^{y}$ satisfying (1.1) may be regarded as a Bessel-generalized shift operator Bessel-generalized shift operator (see $[4,11]$ and $[21])$. We remark that this shift operator is closely connected with the Bessel differential operator. The convolution operator determined by $T^{y}$ is as follows:

$$
(f * \varphi)(x)=\int_{\mathbb{R}_{+}^{n}} f(y) T^{y} \varphi(x) d \mu_{\gamma}(y) .
$$

The convolution (1.3) is known as a $B$-convolution. We note some properties for the $B$ convolution and the Bessel-generalized shift operator:

- If $f(x), \varphi(x) \in \mathcal{C}\left(\mathbb{R}_{+}^{n}\right), \varphi(x)$ is a bounded function, $x>0$, and

$$
\int_{0}^{\infty}|f(x)| d \mu_{\gamma}(x)<\infty
$$

then

$$
\int_{\mathbb{R}_{+}^{n}} T^{y} f(x) \varphi(y) d \mu_{\gamma}(y)=\int_{\mathbb{R}_{+}^{n}} f(y) T^{y} \varphi(x) d \mu_{\gamma}(y) .
$$

- From the above result, we have the following equality for $\varphi(x)=1$ :

$$
\int_{\mathbb{R}_{+}^{n}} T^{y} f(x) d \mu_{\gamma}(y)=\int_{\mathbb{R}_{+}^{n}} f(y) d \mu_{\gamma}(y) .
$$

- $(f * \varphi)(x)=(\varphi * f)(x)$.

The Fourier-Bessel transform is defined and invertible on functions $\varphi \in \mathcal{S}\left(\mathbb{R}_{+}^{n}\right)$,

$$
\begin{aligned}
& {\left[F_{\mathrm{B}} \varphi\right](y)=c_{\gamma} \int_{R_{n}^{+}} \varphi(x) \prod_{i=1}^{n} j_{\gamma_{i}-\frac{1}{2}}\left(x_{i} y_{i}\right) d \mu_{\gamma}(x),} \\
& {\left[F_{\mathrm{B}}^{-1} \varphi\right](x)=\int_{R_{n}^{+}} \varphi(y) \prod_{i=1}^{n} j_{\gamma_{i}-\frac{1}{2}}\left(x_{i} y_{i}\right) d \mu_{\gamma}(y),}
\end{aligned}
$$


where $c_{\gamma}=\prod_{i=1}^{n}\left[2^{\gamma_{i}-\frac{1}{2}} \Gamma\left(\gamma_{i}+\frac{1}{2}\right)\right]^{-1}$ and $j_{\gamma_{i}-\frac{1}{2}}$ is the normalized Bessel function related to the Bessel function of the first kind by the formula $j_{\gamma}(r)=2^{\gamma} \Gamma(\gamma+1) J_{\gamma}(r) r^{-\gamma}$ [11]. However, the following equality for a Fourier-Bessel transformation is true:

$$
F_{\mathrm{B}}(f * \varphi)(x)=F_{\mathrm{B}} f(x) F_{\mathrm{B}} \varphi(x)
$$

(see $[22-28])$.

\section{The high order Riesz-Bessel transforms $R_{\mathrm{B}}$ associated with Bessel-generalized shift operator}

In this section, we consider a Bessel-generalized shift operator related to the multidimensional Bessel differential operator. Then we give the Fourier-Bessel transformation of a homogeneous polynomial which obeys the Bessel equations. Finally, we define the high order Riesz-Bessel transforms related to Bessel-generalized shift operator and so we show that high order Riesz-Bessel transforms obey the condition of classical Riesz transforms, that is, these operators extend to high order Riesz-Bessel transforms [29].

It follows from the general theory of singular integrals that Riesz transforms are bounded on $L_{p, \gamma}\left(\mathbb{R}^{n}, d \mu_{\gamma}(x)\right)$ for all $1<p<\infty$. In this paper we extend this result to the context of Bessel theory where a similar operator is already defined. It has been noted that the difficulty arises in the application of the classical $L_{p}$-theory of Calderon-Zygmund, since Riesz transforms are singular integral operators. In this paper we describe how this theory can be adapted in a Bessel setting and give an $L_{p, \gamma}$-result for high order Riesz transforms for all $1<p<\infty$.

We have $\Omega(y)=P_{k}(y)|y|^{-k}, K(y)=\Omega(y)|x|^{-n-2|\gamma|}$, the $P_{k}$ range over the homogeneous harmonic polynomials the latter arise in special case $k=1$. Those for $k>1$, we call the high order Riesz-Bessel transform where we refer to $k$ as the degree of the high order Riesz Bessel transform [29]. They can also be characterized by their invariance properties.

Let $P_{k}$ be homogeneous polynomial of degree $k$ in $\mathbb{R}_{+}^{n}$. We shall say that $P$ is elliptic if $P(x)$ vanishes only at the origin. For any polynomial $P$ we consider also its corresponding differential polynomial. Thus if $P(x)=\sum a_{\alpha} x^{\alpha}$ we write $P\left(\frac{\partial}{\partial x}\right)=\sum a_{\alpha}\left(\frac{\partial}{\partial x}\right)^{\alpha}$, where $\left(\frac{\partial}{\partial x}\right)^{\alpha}=$ $\left(\frac{\partial}{\partial x_{1}}\right)^{\alpha_{1}} \cdots\left(\frac{\partial}{\partial x_{n}}\right)^{\alpha_{n}}$ and with the monomials $x^{\alpha}=x_{1}^{\alpha_{1}} \cdots x_{n}^{\alpha_{n}}$ (see [30]).

Theorem 2.1 Suppose that $P_{k}(x)$ is a homogeneous polynomial of degree $k$ and satisfies for Bessel operator $\mathrm{B}\left[P_{k}(x)\right]=0$ then we have

$$
F_{\mathrm{B}}\left[P_{k}(x) e^{-|x|^{2}}\right](y)=2^{-\left(|\gamma|+k+\frac{n}{2}\right)} i^{k} P_{k}(y) e^{\frac{-|y|^{2}}{4}} .
$$

Lemma 2.2 Let $\theta=\left(\theta_{1}, \theta_{2}, \ldots, \theta_{n}\right)$. Suppose that

$$
\int_{S_{+}^{n}} f(\theta) d \mu_{\gamma}(\theta) d S=0
$$

and $\varphi$ is of Schwartz class $\mathcal{S}\left(\mathbb{R}_{+}^{n}\right)$ then we have the identity

$$
\lim _{\varepsilon \rightarrow 0} \int_{\mathbb{R}^{n}} \frac{f\left(\frac{x}{|x|}\right)}{|x|^{n+2|\gamma|-\varepsilon}} \varphi(x) d \mu_{\gamma}(x)=\lim _{\varepsilon \rightarrow 0} \int_{|x|>\varepsilon} \frac{f\left(\frac{x}{|x|}\right)}{|x|^{n+2|\gamma|}} \varphi(x) d \mu_{\gamma}(x) .
$$


Proof The proof follows immediately from the representation

$$
\begin{aligned}
\int_{\mathbb{R}_{+}^{n}} \frac{f\left(\frac{x}{|x|}\right)}{|x|^{n+2|\gamma|-\varepsilon}} \varphi(x) d \mu_{\gamma}(x)= & \int_{|x| \leq 1} \frac{f\left(\frac{x}{|x|}\right)}{|x|^{n+2|\gamma|-\varepsilon}}[\varphi(x)-\varphi(0)] d \mu_{\gamma}(x) \\
& +\int_{|x|>1} \frac{f\left(\frac{x}{|x|}\right)}{|x|^{n+2|\gamma|-\varepsilon}} \varphi(x) d \mu_{\gamma}(x) .
\end{aligned}
$$

The mean value theorem for multidimensional Bessel differential operators is very convenient for obtaining multidimensional singular integral operators generated by a Besselgeneralized shift operator. Therefore, we studied the mean value formula related to the Bessel-generalized shift operator for the solutions of the boundary value problem for the multidimensional Bessel operator $B u=0$.

The Bessel-generalized shift operator is one of the most important generalized shift operator on the half line $\mathbb{R}_{+}=[0,+\infty)$ [11-13]. The Bessel-generalized translation is used while studying various problems connected with Bessel operators. Fourier-Bessel harmonic analysis, i.e. the part of harmonic analysis addressing various problems on Bessel (Hankel) integral transforms, is closely connected with the Bessel-generalized shift operator.

\section{The mean value formula}

In this section, we determine the mean value formula for the Bessel-generalized shift operator. Let $\mathbb{R}^{n}$ be an $n$-dimensional Euclidian space and $x=\left(x_{1}, x_{2}, \ldots, x_{n}\right), \xi=\left(\xi_{1}, \xi_{2}, \ldots, \xi_{n}\right)$ be vectors in $\mathbb{R}^{n}$. Then $x \cdot \xi=x_{1} \xi_{1}+\cdots+x_{n} \xi_{n}$. Denote $\mathbb{R}_{+}^{n}=\left\{x \in \mathbb{R}^{n}: x_{i}>0,1 \leq i \leq n\right\}$ and $S_{+}^{n-1}=\left\{x \in \mathbb{R}_{+}^{n}:|x|=1\right\},|\gamma|=\gamma_{1}+\cdots+\gamma_{n}$. We assume that $D^{+} \subset \mathbb{R}_{+}^{n}$ and $\Omega^{+}$is its boundary. In this paper, we are mainly concerned with the mean value theorem. Now, we relate this concept in the following theorem.

Theorem 3.1 Let $S_{+}^{n-1}$ be a unit sphere centered at the origin, contained in $\mathbb{R}_{+}^{n}$ and $u$ be an even regular solution with respect to $x_{1}, \ldots, x_{n}$ of $\mathrm{B} u=0$. Then the following formula holds:

$$
\int_{S_{+}^{n-1}} u\left(r \theta_{1}, \ldots, r \theta_{n}\right) \prod_{i=1}^{n} \theta_{i}^{2 \gamma_{i}} d S=\frac{\prod_{i=1}^{n} \Gamma\left(\gamma_{i}+\frac{1}{2}\right)}{2^{n-1} \Gamma\left(|\gamma|+\frac{n}{2}\right)} u(0) .
$$

Let $T^{y}$ be the multidimensional Bessel-generalized shift operator and $u$ be an even regular solution of $\mathrm{B} u=0$ at any interior point of the region $D^{+} \subseteq \mathbb{R}_{+}^{n}$. Also, the following formula is valid:

$$
\int_{S_{+}^{n-1}} T^{y} u\left(r \theta_{1}, r \theta_{2}, \ldots, r \theta_{n}\right) \prod_{i=1}^{n} \theta_{i}^{2 \gamma_{i}} d S=\frac{\prod_{i=1}^{n} \Gamma\left(\gamma_{i}+\frac{1}{2}\right)}{2^{n-1} \Gamma\left(|\gamma|+\frac{n}{2}\right)} u(y) .
$$

Proof We assume that the continuity of $u$ and $v$ in the closed region $\Omega^{+} \cup D^{+}$, continuity of the first and second derivatives of $u$ and $v$ in $D^{+}$, together with continuity of the first derivatives of $u$ in $\Omega^{+} \cup D^{+}$and the second derivatives of $u$ in $D^{+}$. In addition, the existence of the integrals over $D^{+}$are assumed in Green's formula. The most important tool for the potential theory is provided by this formula in $n$-dimensional bounded region $D^{+}$with volume element $d g=d x_{1} \cdots d x_{n}$ and its boundary $\Omega^{+}$, which we assume to be piecewise 
smooth; the two functions $u$ and $v$ are related by Green's formula,

$$
\int_{\Omega^{+}}\left(u \frac{\partial v}{\partial n}-v \frac{\partial u}{\partial n}\right) \prod_{i=1}^{n} x_{i}^{2 \gamma_{i}} d \Omega=\iint_{D^{+}}[u \mathrm{~B} v-v \mathrm{~B} u] \prod_{i=1}^{n} x_{i}^{2 \gamma_{i}} d g
$$

Let $P^{\prime}=(0, \ldots, 0)$ be a boundary point of $\Omega^{+}$. We set

$$
v=\left[(n+2|\gamma|-2) r^{n+2|\gamma|-2}\right]^{-1}+w(r)
$$

such that $r^{2}=x_{1}^{2}+x_{2}^{2}+\cdots+x_{n}^{2}$ and $w \in \mathcal{C}^{2}\left(D^{+}\right)$denotes the set of twice continuously differentiable functions on $D^{+}$, also an even function with respect to all $x_{i}$-variables. We apply formula (3.3) to the region $D^{+} \backslash D_{\varepsilon}^{+} \subset D^{+}$, where $D_{\varepsilon}^{+}$is an upper half sphere centered at $P^{\prime}$ of radius $\varepsilon$. Letting $\varepsilon \rightarrow 0$ and considering $\left[(n+2|\gamma|-2) r^{n+2|\gamma|-2}\right]^{-1}$ for the fundamental solution of $B$, we deduce that

$$
\begin{aligned}
& \iint_{D^{+} \backslash D_{\epsilon}^{+}}[u \mathrm{~B} v-v \mathrm{~B} u] \prod_{i=1}^{n} x_{i}^{2 \gamma_{i}} d x \\
& \quad=\int_{\Omega}\left(u \frac{\partial v}{\partial n}-v \frac{\partial u}{\partial n}\right) \prod_{i=1}^{n} x_{i}^{2 \gamma_{i}} d \Omega-\int_{\Omega \epsilon}\left(u \frac{\partial v}{\partial n}-v \frac{\partial u}{\partial n}\right) \prod_{i=1}^{n} x_{i}^{2 \gamma_{i}} d \Omega .
\end{aligned}
$$

Hence, let us write the function $v$ in (3.4), then we have

$$
\begin{gathered}
\iint_{D^{+}}[u \mathrm{~B} v-v \mathrm{~B} u] \prod_{i=1}^{n} x_{i}^{2 \gamma_{i}} d x-\iint_{D_{\epsilon}^{+}}[u \mathrm{~B} w-w \mathrm{~B} u] \prod_{i=1}^{n} x_{i}^{2 \gamma_{i}} d x \\
-\iint_{D_{\epsilon}^{+}}\left[(n+2|\gamma|-2) r^{n+2|\gamma|-2}\right]^{-1} \mathrm{~B} u \prod_{i=1}^{n} x_{i}^{2 \gamma_{i}} d x \\
=\int_{\Omega}\left(u \frac{\partial v}{\partial n}-v \frac{\partial u}{\partial n}\right) \prod_{i=1}^{n} x_{i}^{2 \gamma_{i}} d \Omega+\int_{\Omega_{\epsilon}} u \frac{1}{r^{n+2|\gamma|-1}} \prod_{i=1}^{n} x_{i}^{2 \gamma_{i}} d \Omega \\
-\int_{\Omega_{\epsilon}}\left(u \frac{\partial w}{\partial n}-w \frac{\partial u}{\partial n}\right) \prod_{i=1}^{n} x_{i}^{2 \gamma_{i}} d \Omega \\
-\int_{\Omega_{\epsilon}}\left[(n+2|\gamma|-2) r^{n+2|\gamma|-2}\right]^{-1} \frac{\partial u}{\partial n} \prod_{i=1}^{n} x_{i}^{2 \gamma_{i}} d \Omega
\end{gathered}
$$

and

$$
\begin{aligned}
& \iint_{D^{+}}[u \mathrm{~B} v-v \mathrm{~B} u] \prod_{i=1}^{n} x_{i}^{2 \gamma_{i}} d g \\
& =\int_{\Omega}\left(u \frac{\partial v}{\partial n}-v \frac{\partial u}{\partial n}\right) \prod_{i=1}^{n} x_{i}^{2 \gamma_{i}} d \Omega \\
& \quad+\left[(n+2|\gamma|-2) r^{n+2|\gamma|-2}\right]^{-1} \iint_{D_{\epsilon}^{+}} \mathrm{B} u \prod_{i=1}^{n} x_{i}^{2 \gamma_{i}} d g \\
& \quad-\left[(n+2|\gamma|-2) r^{n+2|\gamma|-2}\right]^{-1} \int_{\Omega_{\epsilon}} \frac{\partial u}{\partial n} \prod_{i=1}^{n} x_{i}^{2 \gamma_{i}} d \Omega+\int_{\Omega_{\epsilon}} u \frac{1}{r^{n+2|\gamma|-1}} \prod_{i=1}^{n} x_{i}^{2 \gamma_{i}} d \Omega .
\end{aligned}
$$


Therefore, we get

$$
\begin{aligned}
\iint_{D^{+}}[u \mathrm{~B} v-v \mathrm{~B} u] \prod_{i=1}^{n} x_{i}^{2 \gamma_{i}} d g= & \int_{\Omega}\left(u \frac{\partial v}{\partial n}-v \frac{\partial u}{\partial n}\right) \prod_{i=1}^{n} x_{i}^{2 \gamma_{i}} d \Omega \\
& +\int_{\Omega_{\epsilon}} u \frac{1}{r^{n+2|\gamma|-1}} \prod_{i=1}^{n} x_{i}^{2 \gamma_{i}} d \Omega .
\end{aligned}
$$

Considering $\mathrm{B} v=\mathrm{B} w$, consequently, we have

$$
\begin{aligned}
\iint_{D^{+}}[u \mathrm{~B} v-v \mathrm{~B} u] \prod_{i=1}^{n} x_{i}^{2 \gamma_{i}} d g= & \frac{\prod_{i=1}^{n} \Gamma\left(\gamma_{i}+\frac{1}{2}\right)}{2^{n-1} \Gamma\left(|\gamma|+\frac{n}{2}\right)} u(0) \\
& +\int_{\Omega}\left(u \frac{\partial v}{\partial n}-v \frac{\partial u}{\partial n}\right) \prod_{i=1}^{n} x_{i}^{2 \gamma_{i}} d \Omega .
\end{aligned}
$$

Here,

$$
\int_{\Omega_{\epsilon}} \frac{1}{r^{n+2|\gamma|-1}} \prod_{i=1}^{n} x_{i}^{2 \gamma_{i}} d \Omega=\frac{\prod_{i=1}^{n} \Gamma\left(\gamma_{i}+\frac{1}{2}\right)}{2^{n-1} \Gamma\left(|\gamma|+\frac{n}{2}\right)}=m\left(\Omega_{\epsilon}\right)
$$

and

$$
\lim _{\epsilon \rightarrow 0}\left[\int_{\Omega_{\epsilon}} u \frac{1}{r^{n+2|\gamma|-1}} \prod_{i=1}^{n} x_{i}^{2 \gamma_{i}} d \Omega\right]=m\left(\Omega_{\epsilon}\right) u(0) .
$$

Let $K_{R}^{+}$be a half sphere of radius $R$ centered at $P^{\prime}$ contained in $D^{+}$and $W_{R}^{+}$be the surface of $K_{R}^{+}$. Suppose that

$$
v=(n+2|\gamma|-2)^{-1}\left[r^{-(n+2|\gamma|-2)}-R^{-(n+2|\gamma|-2)}\right] .
$$

Under our assumptions, we can rewrite the equality (3.6) as

$$
\begin{gathered}
\int_{K_{R}^{+}}\left\{u \mathrm{~B}(n+2|\gamma|-2)^{-1}\left[r^{-(n+2|\gamma|-2)}-R^{-(n+2|\gamma|-2)}\right]-v \mathrm{~B} u\right\} \prod_{i=1}^{n} x_{i}^{2 \gamma_{i}} d g \\
=\int_{W_{R}^{+}}\left(u \frac{\partial v}{\partial n}-v \frac{\partial u}{\partial n}\right) \prod_{i=1}^{n} x_{i}^{2 \gamma_{i}} d w+\frac{\prod_{i=1}^{n} \Gamma\left(\gamma_{i}+\frac{1}{2}\right)}{2^{n-1} \Gamma\left(|\gamma|+\frac{n}{2}\right)} u(0) .
\end{gathered}
$$

Since $R$ is constant and $\left[(n+2|\gamma|-2) r^{n+2|\gamma|-2}\right]^{-1}$ for the fundamental solution of $\mathrm{B}$, hence we have

$$
\begin{aligned}
-\int_{K_{R}^{+}} v \mathrm{~B} u \prod_{i=1}^{n} x_{i}^{2 \gamma_{i}} d g= & \frac{\prod_{i=1}^{n} \Gamma\left(\gamma_{i}+\frac{1}{2}\right)}{2^{n-1} \Gamma\left(|\gamma|+\frac{n}{2}\right)} u(0) \\
& +\int_{W_{R}^{+}}\left\{\left[u \frac{\partial(n+2|\gamma|-2)^{-1}}{\partial n}-(n+2|\gamma|-2)^{-1}\right]\right. \\
& \left.\times\left[r^{-(n+2|\gamma|-2)}-R^{-(n+2|\gamma|-2)}\right] \frac{\partial u}{\partial n}\right\} \prod_{i=1}^{n} x_{i}^{2 \gamma_{i}} d w
\end{aligned}
$$




$$
\begin{aligned}
= & \frac{\prod_{i=1}^{n} \Gamma\left(\gamma_{i}+\frac{1}{2}\right)}{2^{n-1} \Gamma\left(|\gamma|+\frac{n}{2}\right)} u(0) \\
& +\int_{W_{R}^{+}} u \frac{\partial(n+2|\gamma|-2)^{-1} r^{-(n+2|\gamma|-2)}}{\partial n} \prod_{i=1}^{n} x_{i}^{2 \gamma_{i}} d w \\
= & \frac{\prod_{i=1}^{n} \Gamma\left(\gamma_{i}+\frac{1}{2}\right)}{2^{n-1} \Gamma\left(|\gamma|+\frac{n}{2}\right)} u(0)-\frac{1}{R^{n+2|\gamma|-1}} \int_{W_{R}^{+}} u \prod_{i=1}^{n} x_{i}^{2 \gamma_{i}} d w
\end{aligned}
$$

and

$$
\begin{gathered}
\frac{2^{n-1} \Gamma\left(|\gamma|+\frac{n}{2}\right)}{\prod_{i=1}^{n} \Gamma\left(\gamma_{i}+\frac{1}{2}\right) R^{n+2|\gamma|-1}} \int_{W_{R}^{+}} u\left(x_{1}, \ldots, x_{n}\right) \prod_{i=1}^{n} x_{i}^{2 \gamma_{i}} d w \\
=u(0)+\frac{2^{n-1} \Gamma\left(|\gamma|+\frac{n}{2}\right)}{\prod_{i=1}^{n} \Gamma\left(\gamma_{i}+\frac{1}{2}\right)} \int_{K_{R}^{+}} v \mathrm{~B} u \prod_{i=1}^{n} x_{i}^{2 \gamma_{i}} d g .
\end{gathered}
$$

Choose the function $v$ such that

$$
v=c \frac{2^{n-1} \Gamma\left(|\gamma|+\frac{n}{2}\right)}{\prod_{i=1}^{n} \Gamma\left(\gamma_{i}+\frac{1}{2}\right)}\left[(n+2|\gamma|-2) r^{n+2|\gamma|-2}\right]^{-1}+w(r)
$$

where $w(r) \in \mathcal{C}^{(2)}\left(K_{R}^{+}\right)$, also an even function with respect to all variables and $0<r \leq R$. Then, under the initial conditions

$$
\left.v\right|_{W_{R}^{+}}=\left.\frac{\partial v}{\partial r}\right|_{W_{R}^{+}}=0
$$

we obtain

$$
\begin{aligned}
\int_{K_{R}^{+}}[u \mathrm{~B} v-v \mathrm{~B} u] \prod_{i=1}^{n} x_{i}^{2 \gamma_{i}} d g & =\int_{W_{R}^{+}}\left(u \frac{\partial v}{\partial n}-v \frac{\partial u}{\partial n}\right) \prod_{i=1}^{n} x_{i}^{2 \gamma_{i}} d w \\
& =\int_{W_{R}^{+}} u \frac{\partial v}{\partial n} \prod_{i=1}^{n} x_{i}^{2 \gamma_{i}} d w \\
& =c \frac{2^{n-1} \Gamma\left(|\gamma|+\frac{n}{2}\right)}{\prod_{i=1}^{n} \Gamma\left(\gamma_{i}+\frac{1}{2}\right)} \int_{W_{R}^{+}} u \frac{1}{r^{n+2|\gamma|-1}} \prod_{i=1}^{n} x_{i}^{2 \gamma_{i}} d w \\
& =c u(0) .
\end{aligned}
$$

Since (3.11) is true for all $u$, we can take an arbitrary function that is even with respect to all variables in $\mathcal{C}^{(2)}\left(K_{R}^{+}\right)$. Therefore, we assume that $u \in \mathcal{C}^{(2 m+2)}\left(K_{R}^{+}\right)$. For $\eta \leq m$, we have the following identity:

$$
c \mathrm{~B}^{\eta} u(0)=\int_{K_{R}^{+}}\left[\mathrm{B}^{\eta} u \mathrm{~B} v-v \mathrm{~B}^{\eta+1} u\right] \prod_{i=1}^{n} x_{i}^{2 \gamma_{i}} d g
$$

where $\mathrm{B}^{\eta}$ denotes the $\eta$ th order of $\mathrm{B}$, that is, $\mathrm{B}^{1} u=\mathrm{B} u, \mathrm{~B}^{2} u=\mathrm{BB} u$, etc. Now, let us define $\left\{v_{\eta}\right\}$ as a sequence of functions of the type (3.9). Then the following differential equation 
can be written:

$$
\mathrm{B} v_{\eta+1}=v_{\eta+1}^{\prime \prime}+\frac{(n+2|\gamma|-1)}{r} v_{\eta+1}^{\prime}=v_{\eta} \quad(\eta=0,1,2, \ldots, m) .
$$

By the initial conditions (3.10) and the function

$$
v_{0}=\frac{2^{n-1} \Gamma\left(|\gamma|+\frac{n}{2}\right)}{\prod_{i=1}^{n} \Gamma\left(\gamma_{i}+\frac{1}{2}\right)}(n+2|\gamma|-2)^{-1}\left[r^{-(n+2|\gamma|-2)}-R^{-(n+2|\gamma|-2)}\right],
$$

it can be seen that the solution of (3.13) is as follows:

$$
v_{\eta+1}=\left[(n+2|\gamma|-2) r^{n+2|\gamma|-2}\right]^{-1} \int_{r}^{R} \rho v_{\eta}(\rho)\left[\rho^{n+2|\gamma|-2}-r^{n+2|\gamma|-2}\right] d \rho .
$$

Under the initial conditions, for each $\eta=0,1,2, \ldots$, the solutions $v_{0}, v_{1}, \ldots$ corresponding to the constants $c_{0}, c_{1}, \ldots$ can easily be found and obtained as follows:

$$
c_{\eta}=\left(\frac{R}{2}\right)^{2 \eta} \frac{\Gamma\left(|\gamma|+\frac{n}{2}\right)}{\eta ! \Gamma\left(\eta+|\gamma|+\frac{n}{2}\right)} \quad(\eta=0,1,2, \ldots) .
$$

Replacing $v$ by $v_{\eta}$ in (3.12), we see that

$$
c_{\eta} \mathrm{B}^{\eta} u(0)=\int_{K_{R}^{+}}\left[\mathrm{B}^{\eta} u \mathrm{~B} v_{\eta}-v_{\eta} \mathrm{B}^{\eta+1} u\right] \prod_{i=1}^{n} x_{i}^{2 \gamma_{i}} d g
$$

Since $B v_{\eta}=v_{\eta-1}$, we obtain

$$
c_{\eta} \mathrm{B}^{\eta} u(0)=\int_{K_{R}^{+}}\left[v_{\eta-1} \mathrm{~B}^{\eta} u-v_{\eta} \mathrm{B}^{\eta+1} u\right] \prod_{i=1}^{n} x_{i}^{2 \gamma_{i}} d g
$$

Summing all the equations corresponding to $\eta=1,2, \ldots, m$, then we conclude

$$
\sum_{\eta=1}^{m} c_{\eta} \mathrm{B}^{\eta} u(0)=\int_{K_{R}^{+}}\left[v_{0} \mathrm{~B} u-v_{m} \mathrm{~B}^{m+1} u\right] \prod_{i=1}^{n} x_{i}^{2 \gamma_{i}} d g .
$$

By (3.8) and the function $v_{0}$, then we get

$$
\int_{K_{R}^{+}} v_{0} \mathrm{~B} u \prod_{i=1}^{n} x_{i}^{2 \gamma_{i}} d g=\sum_{\eta=1}^{m} c_{\eta} \mathrm{B}^{\eta} u(0)+\int_{K_{R}^{+}} v_{m} \mathrm{~B}^{m+1} u \prod_{i=1}^{n} x_{i}^{2 \gamma_{i}} d g
$$

and

$$
\begin{aligned}
& \frac{2^{n-1} \Gamma\left(|\gamma|+\frac{n}{2}\right)}{\prod_{i=1}^{n} \Gamma\left(\gamma_{i}+\frac{1}{2}\right)} \int_{K_{R}^{+}}(n+2|\gamma|-2)^{-1}\left[r^{-(n+2|\gamma|-2)}-R^{-(n+2|\gamma|-2)}\right] \mathrm{B} u \prod_{i=1}^{n} x_{i}^{2 \gamma_{i}} d g \\
& \quad=\sum_{\eta=1}^{m}\left(\frac{R}{2}\right)^{2 \eta} \frac{\Gamma\left(|\gamma|+\frac{n}{2}\right)}{\eta ! \Gamma\left(\eta+|\gamma|+\frac{n}{2}\right)} \mathrm{B}^{\eta} u(0)+\int_{K_{R}^{+}} v_{m} \mathrm{~B}^{m+1} u \prod_{i=1}^{n} x_{i}^{2 \gamma_{i}} d g .
\end{aligned}
$$


By the function $v$ in (3.7), we get

$$
\begin{aligned}
\frac{2^{n-1} \Gamma\left(|\gamma|+\frac{n}{2}\right)}{\prod_{i=1}^{n} \Gamma\left(\gamma_{i}+\frac{1}{2}\right)} \int_{K_{R}^{+}} v \mathrm{~B} u \prod_{i=1}^{n} x_{i}^{2 \gamma_{i}} d g= & \sum_{\eta=1}^{m}\left(\frac{R}{2}\right)^{2 \eta} \frac{\Gamma\left(|\gamma|+\frac{n}{2}\right)}{\eta ! \Gamma\left(\eta+|\gamma|+\frac{n}{2}\right)} \mathrm{B}^{\eta} u(0) \\
& +\int_{K_{R}^{+}} v_{m} \mathrm{~B}^{m+1} u \prod_{i=1}^{n} x_{i}^{2 \gamma_{i}} d g
\end{aligned}
$$

and from (3.8)

$$
\begin{aligned}
& {\left[c_{n, \gamma} R^{n+2|\gamma|-1}\right]^{-1} \int_{W_{R}^{+}} u(x) \prod_{i=1}^{n} x_{i}^{2 \gamma_{i}} d w} \\
& \quad=u(0)+\sum_{\eta=1}^{m}\left(\frac{R}{2}\right)^{2 \eta} \frac{\Gamma\left(|\gamma|+\frac{n}{2}\right)}{\eta ! \Gamma\left(\eta+|\gamma|+\frac{n}{2}\right)} \mathrm{B}^{\eta} u(0)+\int_{K_{R}^{+}} v_{m} \mathrm{~B}^{m+1} u \prod_{i=1}^{n} x_{i}^{2 \gamma_{i}} d g .
\end{aligned}
$$

Thus we have

$$
\begin{aligned}
& {\left[c_{n, \gamma} R^{n+2|\gamma|-1}\right]^{-1} \int_{W_{R}^{+}} u(x) \prod_{i=1}^{n} x_{i}^{2 \gamma_{i}} d w} \\
& \quad=\sum_{\eta=0}^{m}\left(\frac{R}{2}\right)^{2 \eta} \frac{\Gamma\left(|\gamma|+\frac{n}{2}\right)}{\eta ! \Gamma\left(\eta+|\gamma|+\frac{n}{2}\right)} \mathrm{B}^{\eta} u(0)+\int_{K_{R}^{+}} v_{m} \mathrm{~B}^{m+1} u \prod_{i=1}^{n} x_{i}^{2 \gamma_{i}} d g,
\end{aligned}
$$

where $c_{0}=1, \mathrm{~B}^{0} u_{0}=u_{0}$ and $c_{n, \gamma}=\frac{\prod_{i=1}^{n} \Gamma\left(\gamma_{i}+\frac{1}{2}\right)}{2^{n-1} \Gamma\left(|\gamma|+\frac{n}{2}\right)}$. This formula is valid for all arbitrary function $u \in K_{R}^{+} \subseteq D^{+}$which is a continuously differentiable function of order $(2 m+2)$. Let us establish the equality (3.8) with respect to $T^{y} u(x)$; we get

$$
\begin{aligned}
& \frac{2^{n-1} \Gamma\left(|\gamma|+\frac{n}{2}\right)}{\prod_{i=1}^{n} \Gamma\left(\gamma_{i}+\frac{1}{2}\right) R^{n+2|\gamma|-1}} \int_{W_{R}^{+}} T^{y} u(x) \prod_{i=1}^{n} x_{i}^{2 \gamma_{i}} d w \\
& \quad=u(y)+\frac{2^{n-1} \Gamma\left(|\gamma|+\frac{n}{2}\right)}{\prod_{i=1}^{n} \Gamma\left(\gamma_{i}+\frac{1}{2}\right)} \int_{K_{R}^{+}} v \mathrm{~B} T^{y} u \prod_{i=1}^{n} x_{i}^{2 \gamma_{i}} d g .
\end{aligned}
$$

Setting $v=v_{\eta}$ and $u(x)=T^{y} u(x)$ in (3.12), then we obtain

$$
\begin{aligned}
& c \mathrm{~B}^{\eta} u(y)=\int_{K_{R}^{+}}\left[\mathrm{B}^{\eta} T^{y} u(x) \mathrm{B} v-v \mathrm{~B}^{\eta+1} T^{y} u(x)\right] \prod_{i=1}^{n} x_{i}^{2 \gamma_{i}} d g, \\
& c_{\eta} \mathrm{B}^{\eta} u(y)=\int_{K_{R}^{+}}\left[\mathrm{B}^{\eta} T^{y} u(x) \mathrm{B} v_{\eta}-v_{\eta} \mathrm{B}^{\eta+1} T^{y} u(x)\right] \prod_{i=1}^{n} x_{i}^{2 \gamma_{i}} d g .
\end{aligned}
$$

If we sum all the equations corresponding to $\eta=(1,2, \ldots, m)$, we conclude

$$
\sum_{\eta=1}^{m} c_{\eta} \mathrm{B}^{\eta} u(y)=\int_{K_{R}^{+}}\left[v_{0} \mathrm{~B} T^{y} u-v_{m} \mathrm{~B}^{m+1} T^{y} u\right] \prod_{i=1}^{n} x_{i}^{2 \gamma_{i}} d g .
$$


By (3.8) and the function $v_{0}$, we get

$$
\begin{aligned}
\int_{K_{R}^{+}} v_{0} \mathrm{~B} T^{y} u(x) \prod_{i=1}^{n} x_{i}^{2 \gamma_{i}} d g= & \sum_{\eta=1}^{m}\left(\frac{R}{2}\right)^{2 \eta} \frac{\Gamma\left(|\gamma|+\frac{n}{2}\right)}{\eta ! \Gamma\left(\eta+|\gamma|+\frac{n}{2}\right)} \mathrm{B}^{\eta} u(y) \\
& +\int_{W_{R}^{+}} v_{m} \mathrm{~B}^{m} T^{y} u \prod_{i=1}^{n} x_{i}^{2 \gamma_{i}} d w
\end{aligned}
$$

and

$$
\begin{aligned}
& \frac{2^{n-1} \Gamma\left(|\gamma|+\frac{n}{2}\right)}{\prod_{i=1}^{n} \Gamma\left(\gamma_{i}+\frac{1}{2}\right) R^{n+2|\gamma|-1}} \int_{K_{R}^{+}} v \mathrm{~B}^{y} u(x) \prod_{i=1}^{n} x_{i}^{2 \gamma_{i}} d g \\
& =\sum_{\eta=1}^{m}\left(\frac{R}{2}\right)^{2 \eta} \frac{\Gamma\left(|\gamma|+\frac{n}{2}\right)}{\eta ! \Gamma\left(\eta+|\gamma|+\frac{n}{2}\right)} \mathrm{B}^{\eta} u(y)+\int_{K_{R}^{+}} v_{m} \mathrm{~B}^{m+1} T^{y} u(x) \prod_{i=1}^{n} x_{i}^{2 \gamma_{i}} d g, \\
& {\left[c_{n, \gamma} R^{n+2|\gamma|-1}\right]^{-1} \int_{W_{R}^{+}} T^{y} u(x) \prod_{i=1}^{n} x_{i}^{2 \gamma_{i}} d w_{R}} \\
& =u(y)+\sum_{\eta=1}^{m}\left(\frac{R}{2}\right)^{2 \eta} \frac{\Gamma\left(|\gamma|+\frac{n}{2}\right)}{\eta ! \Gamma\left(\eta+|\gamma|+\frac{n}{2}\right)} \mathrm{B}^{\eta} u(y) \\
& +\int_{K_{R}^{+}} v_{m} \mathrm{~B}^{m+1} T^{y} u(x) \prod_{i=1}^{n} x_{i}^{2 \gamma_{i}} d g,
\end{aligned}
$$

and

$$
\begin{aligned}
& {\left[c_{n, \gamma} R^{n+2|\gamma|-1}\right]^{-1} \int_{W_{R}^{+}} T^{y} u(x) \prod_{i=1}^{n} x_{i}^{2 \gamma_{i}} d w_{R}} \\
& \quad=\sum_{\eta=0}^{m}\left(\frac{R}{2}\right)^{2 \eta} \frac{\Gamma\left(|\gamma|+\frac{n}{2}\right)}{\eta ! \Gamma\left(\eta+|\gamma|+\frac{n}{2}\right)} \mathrm{B}^{\eta} u(y)+\int_{K_{R}^{+}} v_{m} \mathrm{~B}^{m+1} T^{y} u(x) \prod_{i=1}^{n} x_{i}^{2 \gamma_{i}} d g,
\end{aligned}
$$

where $\mathrm{B}^{0} u=u$ and $c_{0}=1$. We note that the second integral on the right-hand side in (3.21) tends to zero for $m \rightarrow \infty$; then we have the result

$$
\begin{aligned}
& \frac{2^{n-1} \Gamma\left(|\gamma|+\frac{n}{2}\right)}{\prod_{i=1}^{n} \Gamma\left(\gamma_{i}+\frac{1}{2}\right) R^{n+2|\gamma|-1}} \int_{S_{n-1}^{+}} T^{y} u\left(R \theta_{1}, \ldots, R \theta_{n}\right) R^{2|\gamma|} \prod_{i=1}^{n} \theta_{i}^{2 \gamma_{i}} R^{n-1} d S \\
& =\sum_{\eta=0}^{\infty}\left(\frac{R}{2}\right)^{2 \eta} \frac{\Gamma\left(|\gamma|+\frac{n}{2}\right)}{\eta ! \Gamma\left(\eta+|\gamma|+\frac{n}{2}\right)} \mathrm{B}^{\eta} u(y),
\end{aligned}
$$

where $x=R \theta$ and $d w_{R}=R^{n-1} d S$. Hence

$$
\int_{S_{+}^{n-1}} T^{y} u\left(R \theta_{1}, R \theta_{2}, \ldots, R \theta_{n}\right) \prod_{i=1}^{n} \theta_{i}^{2 \gamma_{i}} d S=\frac{\prod_{i=1}^{n} \Gamma\left(\gamma_{i}+\frac{1}{2}\right)}{2^{n-1} \Gamma\left(|\gamma|+\frac{n}{2}\right)} u(y) .
$$

Thus the proof is completed. 
Proof of Theorem 2.1 We shall need the Fourier-Bessel transforms of the function $e^{-\alpha|x|^{2}}$. The identity to be proved can be rewritten as

$$
F_{\mathrm{B}}\left(e^{-\alpha|x|^{2}}\right)(y)=c_{\gamma} \int_{\mathbb{R}_{+}^{+}} e^{-\alpha|x|^{2}} \prod_{i=1}^{n} j_{\gamma_{i}-\frac{1}{2}}\left(x_{i} y_{i}\right) d \mu_{\gamma}(x) .
$$

Let $\gamma>-1, \alpha>0$ and $J_{\gamma}(b r)$ be a Bessel function. We recall that

$$
\int_{0}^{\infty} e^{-\alpha r^{2}} r^{\gamma+1} J_{\gamma}(b r) d r=b^{\gamma}(2 \alpha)^{-\gamma-1} e^{\frac{-b^{2}}{4 \alpha}},
$$

then we get

$$
\begin{aligned}
& J_{\gamma_{1}-\frac{1}{2}}\left(x_{1} y_{1}\right)=\left[2^{\gamma_{1}-\frac{1}{2}} \Gamma\left(\gamma_{1}+\frac{1}{2}\right)\right]^{-1}\left(x_{1} y_{1}\right)^{\gamma_{1}-\frac{1}{2}} j_{\gamma_{1}-\frac{1}{2}}\left(x_{1} y_{1}\right), \\
& \vdots \\
& J_{\gamma_{n}-\frac{1}{2}}\left(x_{n} y_{n}\right)=\left[2^{\gamma_{n}-\frac{1}{2}} \Gamma\left(\gamma_{n}+\frac{1}{2}\right)\right]^{-1}\left(x_{n} y_{n}\right)^{\gamma_{n}-\frac{1}{2}} j_{\gamma_{n}-\frac{1}{2}}\left(x_{n} y_{n}\right),
\end{aligned}
$$

where $J_{\gamma}(r)=\left[2^{\gamma} \Gamma(\gamma+1)\right]^{-1} r^{\gamma} j_{\gamma}(r)$. We may now calculate each of the integrals in (3.22). We have

$$
\begin{aligned}
I_{1} & =\int_{0}^{\infty} e^{-\alpha x_{1}{ }^{2}} j_{\gamma_{1}-\frac{1}{2}}\left(x_{1} y_{1}\right) x_{1}^{2 \gamma_{1}} d x_{1} \\
& =\int_{0}^{\infty} e^{-\alpha r^{2}} J_{\gamma_{1}-\frac{1}{2}}(r s) r^{2 \gamma_{1}} r^{-\gamma_{1}+\frac{1}{2}} s^{-\gamma_{1}+\frac{1}{2}} d r \\
& =2^{\gamma_{1}-\frac{1}{2}} \Gamma\left(\gamma_{1}+\frac{1}{2}\right)(2 \alpha)^{-\gamma_{1}-\frac{1}{2}} e^{-\frac{s^{2}}{4 \alpha}},
\end{aligned}
$$

where $x_{1}=r$ and $y_{1}=s$. In a similar way, we obtain

$$
\begin{aligned}
& I_{2}=\int_{0}^{\infty} e^{-\alpha x_{2}{ }^{2}} j_{\gamma_{2}-\frac{1}{2}}\left(x_{2} y_{2}\right) x_{2}^{2 \gamma_{2}} d x_{2}=\frac{2^{\gamma_{2}-\frac{1}{2}} \Gamma\left(\gamma_{2}+\frac{1}{2}\right)}{(2 \alpha)^{\gamma_{2}+\frac{1}{2}}} e^{-\frac{y_{2}^{2}}{4 \alpha}} \\
& I_{n}=\int_{0}^{\infty} e^{-\alpha x_{n}{ }^{2}} j_{\gamma_{n}-\frac{1}{2}}\left(x_{n} y_{n}\right) x_{2}^{2 \gamma_{n}} d x_{n}=\frac{2^{\gamma_{n}-\frac{1}{2}} \Gamma\left(\gamma_{n}+\frac{1}{2}\right)}{(2 \alpha)^{\gamma_{n}+\frac{1}{2}}} e^{-\frac{y_{n}^{2}}{4 \alpha}}
\end{aligned}
$$

If we write the equalities (3.23)-(3.25) in (3.22) then we have

$$
F_{\mathrm{B}}\left(e^{-\alpha|x|^{2}}\right)(y)=e^{-\frac{|y|^{2}}{4 \alpha}}(2 \alpha)^{\frac{-2|\gamma|-n}{2}} \text {. }
$$

Letting $\alpha \rightarrow 1$ and $y \rightarrow-2 y$, then we get

$$
F_{\mathrm{B}}\left(e^{-\alpha|x|^{2}}\right)(y)=e^{-|y|^{2}} 2^{-n} .
$$

So, we can easily obtain the identity

$$
F_{\mathrm{B}}\left(e^{-|x|^{2}}\right)(y)=c_{\gamma} \int_{\mathbb{R}_{+}^{n}} \prod_{i=1}^{n} j_{\gamma_{i}-\frac{1}{2}}\left(x_{i} 2 y_{i}\right) e^{-|x|^{2}} d \mu_{\gamma}(x) .
$$


By similar arguments, we can see that

$$
\begin{aligned}
& I_{1}^{\prime}=\int_{0}^{\infty} e^{-x_{1}{ }^{2}} j_{\gamma_{1}-\frac{1}{2}}\left(x_{1} 2 y_{1}\right) x_{1}^{2 \gamma_{1}} d x_{1} \\
& I_{1}^{\prime}=\int_{0}^{\infty} e^{-r^{\prime 2}} j_{\gamma_{1}-\frac{1}{2}}\left(2 r^{\prime} s^{\prime}\right) r^{\prime 2 \gamma_{1}} d r^{\prime}
\end{aligned}
$$

Hence, we get

$$
I_{1}^{\prime}=2^{-1} \Gamma\left(\gamma_{1}+\frac{1}{2}\right) e^{-s_{1}^{\prime 2}}
$$

and

$$
I_{n}^{\prime}=\int_{0}^{\infty} e^{-r^{\prime 2}} j_{\gamma_{n}-\frac{1}{2}}\left(2 r^{\prime} s^{\prime}\right) r^{\prime 2 \gamma_{n}} d r^{\prime}=2^{-1} e^{-s_{n}^{\prime 2}} \Gamma\left(\gamma_{n}+\frac{1}{2}\right)
$$

Consequently, we deduce

$$
F_{\mathrm{B}}\left(e^{-\alpha|x|^{2}}\right)(y)=2^{-n} \prod_{i=1}^{n} \Gamma\left(\gamma_{i}+\frac{1}{2}\right) e^{-|y|^{2}} .
$$

When we apply the Bessel differential operator $P_{k}\left(\mathrm{~B}_{t_{1}}, \mathrm{~B}_{t_{2}}, \ldots, \mathrm{B}_{t_{n-1}}, \mathrm{~B}_{t_{n}}\right)$ to both sides of the identity (3.26), then we see

$$
\begin{aligned}
F_{\mathrm{B}}\left[P_{k}(x) e^{-|x|^{2}}\right](t)= & \int_{0}^{\infty} P_{k}(x) e^{-x_{1}{ }^{2}} j_{\gamma_{1}-\frac{1}{2}}\left(2 x_{1} t_{1}\right) x_{1}^{2 \gamma_{1}} \\
& \cdots \int_{0}^{\infty} e^{-x_{n}{ }^{2}} j_{\gamma_{n}-\frac{1}{2}}\left(2 x_{n} t_{n}\right) x_{n}^{2 \gamma_{n}} d x \\
= & Q(t) 2^{-n} \prod_{i=1}^{n} \Gamma\left(\gamma_{i}+\frac{1}{2}\right) e^{-|t|^{2}},
\end{aligned}
$$

where $Q(t)$ is a polynomial. The problem is therefore to show that $Q(t)=P_{k}(i y)$. Now using the identity

$$
j_{\gamma-\frac{1}{2}}(r)=\frac{\Gamma\left(\gamma+\frac{1}{2}\right)}{\Gamma(\gamma) \Gamma\left(\frac{1}{2}\right)} \int_{0}^{\pi} e^{i r \cos \alpha}(\sin \alpha)^{2 \gamma-1} d \alpha
$$

we have

$$
\begin{aligned}
Q(t)= & c_{1} \int_{\mathbb{R}_{+}^{n}} P_{k}(x) e^{|t|^{2}-|x|^{2}} \prod_{i=1}^{n} j_{\gamma_{i}-\frac{1}{2}}\left(2 x_{i} t_{i}\right) d \mu_{\gamma}(x) \\
= & c_{1} \int_{\mathbb{R}_{+}^{n}} P_{k}(x) e^{t_{1}^{2}+\cdots+t_{n}^{2}-\left(x_{1}^{2}+\cdots+x_{n}^{2}\right)} \prod_{i=1}^{n} j_{\gamma_{i}-\frac{1}{2}}\left(2 x_{i} t_{i}\right) d \mu_{\gamma}(x) \\
= & c_{2} \int_{\mathbb{R}_{+}^{n}} P_{k}(x) e^{|t|^{2}-|x|^{2}}\left(\int_{0}^{\pi} e^{2 i x_{1} t_{1} \cos w} \sin ^{2 \gamma_{1}-1} w d w\right. \\
& \left.\cdots \int_{0}^{\pi} e^{2 i x_{n} t_{n} \cos w} \sin ^{2 \gamma_{n}-1} w d w\right) d \mu_{\gamma}(x)
\end{aligned}
$$




$$
\begin{aligned}
= & c_{2} \int_{\mathbb{R}_{+}^{n}} P_{k}(x) d \mu_{\gamma}(x) \int_{0}^{\pi} \cdots \int_{0}^{\pi}\left(e^{t_{1}^{2}-x_{1}^{2}-2 i x_{1} t_{1} \cos w} \sin ^{2 \gamma_{1}-1} w\right) \\
& \cdots\left(e^{t_{n}^{2}-x_{n}^{2}-2 i x_{n} t_{n} \cos w} \sin ^{2 \gamma_{n}-1} w\right) d w=2^{n} \int_{\mathbb{R}_{+}^{n}} P_{k}(x)\left[T^{-i t}\left(e^{-|x|^{2}}\right)\right] d \mu_{\gamma}(x),
\end{aligned}
$$

where $c_{1}=2^{n}\left[\prod_{i=1}^{n} \Gamma\left(\gamma_{i}+\frac{1}{2}\right)\right]^{-1}$ and $c_{2}=\prod_{i=1}^{n} 2^{n} \Gamma\left(\gamma_{i}+\frac{1}{2}\right)\left(\pi^{\frac{n}{2}} \Gamma\left(\gamma_{i}\right)\right)^{-1}$. Replacing $t$ by -it and by the properties of $T^{y}$, we obtain

$$
Q(-i t)=c_{1} \int_{\mathbb{R}_{+}^{n}} T^{-t}\left[P_{k}(x) e^{-|x|^{2}}\right] d \mu_{\gamma}(x) .
$$

Taking the change of variables $x \rightarrow r \theta$ for $0<r<\infty$ and $\theta \in \mathcal{S}_{+}^{n-1}$ and applying the polar coordinates in (3.27), this gives the identity

$$
Q(-i t)=c_{1} \int_{0}^{\infty} r^{2|\gamma|+n-1}\left(\int_{\mathcal{S}_{+}^{n-1}} T^{-t}\left[P_{k}(r \theta)\right] \prod_{i=1}^{n} \theta_{i}^{2 \gamma_{i}}\right) e^{-r^{2}} d r d \theta .
$$

We must calculate the integral $\int_{0}^{\infty} r^{2|\gamma|+n-1} e^{-r^{2}} d r$. Letting $r^{2}=u$, then we get

$$
\int_{0}^{\infty} r^{2|\gamma|+n-1} e^{-r^{2}} d r=\frac{1}{2} \int_{0}^{\infty} u^{|\gamma|+\frac{n}{2}-1} e^{-u} d r=\frac{1}{2} \Gamma\left(|\gamma|+\frac{n}{2}\right) .
$$

Applying Theorem 3.1 to (3.28), we deduce $Q(-i t)=P_{k}(t)$ and

$$
\begin{aligned}
F_{\mathrm{B}}\left[P_{k}(x) e^{-\alpha|x|^{2}}\right](t) & =c_{\gamma} \int_{\mathbb{R}_{+}^{n}} P_{k}(x) e^{-|x|^{2}} \prod_{i=1}^{n} j_{\gamma_{i}-\frac{1}{2}}\left(2 x_{i} t_{i}\right) d \mu_{\gamma}(x) \\
& =2^{-\left(|\gamma|+\frac{n}{2}\right)} P_{k}(i t) e^{-|t|^{2}} .
\end{aligned}
$$

Since $P_{k}$ is homogeneous, we have the identity

$$
F_{\mathrm{B}}\left[P_{k}(x) e^{-|x|^{2}}\right](y)=2^{-\left(|\gamma|+k+\frac{n}{2}\right)} i^{k} P_{k}(y) e^{\frac{-|y|^{2}}{4}},
$$

and so we obtain the desired conclusion.

We come now to what has been our main goal in this paper.

Theorem 3.2 Let $P_{k}$ be homogeneous harmonic polynomial of degree $k$. Then we get

$$
F_{\mathrm{B}}\left[p \cdot v \frac{P_{k}(x)}{|x|^{k+n+2|\gamma|}}\right](y)=2^{\frac{-n-2|\gamma|}{2}} i^{k} \frac{\Gamma\left(\frac{k}{2}\right)}{\Gamma\left(\frac{k+n+2|\gamma|}{2}\right)} \frac{P_{k}(y)}{|y|^{k}} .
$$

Proof Consider the identity

$$
F_{\mathrm{B}}[f(\alpha x)](t)=\alpha^{-n-2|\gamma|} F_{\mathrm{B}}[f(x)]\left(\frac{t}{\alpha}\right)
$$

By (3.29) and Theorem 2.1, we have

$$
F_{\mathrm{B}}\left[P_{k}(x) e^{-\alpha|x|^{2}}\right](y)=(2 \alpha)^{-\left(k+|\gamma|+\frac{n}{2}\right)} i^{k} e^{\frac{-|y|^{2}}{4 \alpha}} P_{k}(y) .
$$


Assume in addition that $\varphi \in \mathcal{S}\left(\mathbb{R}_{+}^{n}\right)$. Then

$$
\int_{\mathbb{R}_{+}^{n}} P_{k}(x) e^{-\alpha|x|^{2}} \varphi(x) d \mu_{\gamma}(x)=(2 \alpha)^{-\left(k+|\gamma|+\frac{n}{2}\right)} i^{k} \int_{\mathbb{R}_{+}^{n}} P_{k}(x) e^{-\frac{|x|^{2}}{4 \alpha}} \varphi(x) d \mu_{\gamma}(x) .
$$

We now integrate both sides of the above with respect to $\alpha$ having multiplied the equation by a suitable power of $\alpha\left(\alpha^{\beta-1}, 2 \beta=k+n+2|\gamma|-\varepsilon\right)$. We obtain

$$
\begin{aligned}
\int_{0}^{\infty}\left[\int_{\mathbb{R}_{+}^{n}} P_{k}(x) e^{-\alpha|x|^{2}} \alpha^{\beta-1} \varphi(x) d \mu_{\gamma}(x)\right] d \alpha= & \int_{0}^{\infty}\left[(2 \alpha)^{-\left(k+|\gamma|+\frac{n}{2}\right)} i^{k} \alpha^{\beta-1}\right] \\
& \times\left[\int_{\mathbb{R}_{+}^{n}} P_{k}(x) e^{-\frac{|x|^{2}}{4 \alpha}} \varphi(x) d \mu_{\gamma}(x)\right] d \alpha .
\end{aligned}
$$

If we use the fact that

$$
\int_{0}^{\infty} e^{-u}\left(\frac{u}{|x|^{2}}\right)^{\beta-1} \frac{d u}{|x|^{2}}=\Gamma(\beta)|x|^{-2 \beta}
$$

we get

$$
\int_{0}^{\infty}\left[\int_{\mathbb{R}_{+}^{n}} P_{k}(x) e^{-\alpha|x|^{2}} \alpha^{\beta-1} \varphi(x) d \mu_{\gamma}(x)\right] d \alpha=\Gamma(\beta) \int_{\mathbb{R}_{+}^{n}} \frac{P_{k}(x)}{|x|^{2 \beta}} \varphi(x) d \mu_{\gamma}(x) .
$$

The corresponding integration for the right side gives

$$
\begin{aligned}
\int_{0}^{\infty} & \left\{(2 \alpha)^{-\left(k+|\gamma|+\frac{n}{2}\right)} i^{k} \alpha^{\beta-1}\left[\int_{\mathbb{R}_{+}^{n}} P_{k}(x) e^{-\frac{|x|^{2}}{4 \alpha}} \varphi(x) d \mu_{\gamma}(x)\right]\right\} d \alpha \\
= & \int_{0}^{\infty} 2^{-\left(k+|\gamma|+\frac{n}{2}\right)} i^{k} \alpha^{-\left(\frac{k+\varepsilon}{2}+1\right)} \int_{\mathbb{R}_{+}^{n}}\left[P_{k}(x) e^{-\frac{|x|^{2}}{4 \alpha}} \varphi(x) d \mu_{\gamma}(x)\right] d \alpha \\
= & 2^{-\left(k+|\gamma|+\frac{n}{2}\right)} \int_{0}^{\infty} \int_{\mathbb{R}_{+}^{n}} \alpha^{\left(\frac{-k-\varepsilon}{2}-1\right)} P_{k}(x) e^{-\frac{|x|^{2}}{4 \alpha}} i^{k} \varphi(x) d \mu_{\gamma}(x) d \alpha .
\end{aligned}
$$

Letting $\alpha \rightarrow \frac{1}{4 \alpha}$, we obtain

$$
\begin{aligned}
& \int_{0}^{\infty}\left\{(2 \alpha)^{-\left(k+|\gamma|+\frac{n}{2}\right)} i^{k} \alpha^{\beta-1}\left[\int_{\mathbb{R}_{+}^{n}} P_{k}(x) e^{-\frac{|x|^{2}}{4 \alpha}} \varphi(x) d \mu_{\gamma}(x)\right]\right\} d \alpha \\
& =2^{-\left(|\gamma|+\frac{n}{2}-\varepsilon\right)} \Gamma\left(\frac{k+\varepsilon}{2}\right) i^{k} \int_{\mathbb{R}_{+}^{n}} \frac{P_{k}(x)}{|x|^{k+\varepsilon}} \varphi(x) d \mu_{\gamma}(x) .
\end{aligned}
$$

Thus (3.30) and (3.31) lead to the identity

$$
\int_{\mathbb{R}_{+}^{n}} \frac{P_{k}(x)}{|x|^{2 \beta}} \varphi(x) d \mu_{\gamma}(x)=2^{-\frac{2|\gamma|+n}{2}+\varepsilon} i^{k} \frac{\Gamma\left(\frac{k+\varepsilon}{2}\right)}{\Gamma(\beta)} \int_{\mathbb{R}_{+}^{n}} \frac{P_{k}(x)}{|x|^{k+\varepsilon}} \varphi(x) d \mu_{\gamma}(x) .
$$

By Lemma 2.2, we have therefore concluded the proof of the theorem. We have

$$
F_{\mathrm{B}}\left[p \cdot v \frac{P_{k}(x)}{|x|^{k+n+2|\gamma|-\varepsilon}}\right](y)=2^{-\frac{2|\gamma|+n}{2}} i^{k} \frac{\Gamma\left(\frac{k}{2}\right)}{\Gamma\left(\frac{k+n+2|\gamma|}{2}\right)} \frac{P_{k}(y)}{|y|^{k}} .
$$


Definition 3.3 Let $T^{y}$ be the Bessel-generalized shift operator and let $f$ be a Schwartz function on $\mathbb{R}_{+}^{n}$. We define the high order Riesz-Bessel transforms $R_{\mathrm{B}}^{(k)}$ of order $k$ with respect to the Bessel-generalized shift operator as

$$
\begin{aligned}
R_{\mathrm{B}}^{(k)}(f)(x) & =c_{k}(n, \gamma)\left[p \cdot v \frac{P_{k}(y)}{|y|^{n+k+2|\gamma|}} * f\right](x) \\
& =c_{k}(n, \gamma) \lim _{\varepsilon \rightarrow 0} \int_{0<\varepsilon<|x|} \frac{P_{k}(y)}{|y|^{k+n+2|\gamma|}} T^{y} f(x) d \mu_{\gamma}(y),
\end{aligned}
$$

where $c_{k}(n, \gamma)=2^{\frac{n+2|\gamma|}{2}} \Gamma\left(\frac{n+k+2|\gamma|}{2}\right)\left[\Gamma\left(\frac{k}{2}\right)\right]^{-1}(k=1,2, \ldots, n)$ and $P_{k}(x)$ is a homogeneous polynomial of degree $k$ in $\mathbb{R}_{+}^{n}$ which satisfies $\mathrm{B} P_{k}=0$.

By Theorem 3.2, we conclude that

$$
F_{\mathrm{B}}\left[R_{\mathrm{B}}^{(k)}(f)\right](\xi)=i^{k} P_{k}(\xi)|\xi|^{-k} F_{\mathrm{B}}[f](\xi) .
$$

One of the important applications of the high order Riesz transforms is that they can be used to mediate between various combinations of partial derivatives of a function. We shall here content ourselves with two very simple illustrations, which examples have an interest on their own and have already the characteristic features of a general type of estimate which can be made in the theory of elliptic differential operators.

Proposition 3.4 Suppose $f$ is a class of $\mathcal{S}\left(\mathbb{R}_{+}^{n}\right)$ and has compact support. Let $\mathrm{B} f$ be the Bessel differential operator. Then we have the a priori bound

$$
\left\|\partial_{x_{i}} \partial_{x_{k}} f\right\|_{p, \gamma} \leq A_{p}\|\mathrm{~B} f\|_{p, \gamma}
$$

with $A_{p}$ independent off .

In (3.32), we may take $k=1$. Then this proposition is an immediate consequence of the $L_{p, \gamma}$ boundedness of the Riesz-Bessel transforms generated by a Bessel-generalized shift operator and the identity

$$
\partial_{x_{i}}\left(\partial_{x_{k}} f\right)=-R_{\mathrm{B}} R_{\mathrm{B}} \mathrm{B} f
$$

To prove (3.33) we use the Fourier-Bessel transform. Thus if $F_{\mathrm{B}}[f](x)$ is the Fourier-Bessel transform of $f$, then the Fourier-Bessel transform of $\partial_{x_{i}} f$ is

$$
F_{\mathrm{B}}\left[\partial_{x_{i}} f\right](y)=-x_{i} F_{\mathrm{B}}[f](y)
$$

and

$$
F_{\mathrm{B}}[\mathrm{B} f](y)=-|x|^{2} F_{\mathrm{B}}[f](y),
$$

and so

$$
F_{\mathrm{B}}\left[\partial_{x_{i}}\left(\partial_{x_{k}} f\right)\right](y)=-\left(\frac{x_{i}}{|x|}\right)\left(\frac{x_{k}}{|x|}\right)|x|^{2} F_{\mathrm{B}}[f](y)=-F_{\mathrm{B}}\left[R_{\mathrm{B}} R_{\mathrm{B}} \mathrm{B} f\right](y),
$$


which gives (3.33). Thus we have

$$
\left\|\partial_{x_{i}}\left(\partial_{x_{k}} f\right)\right\|_{p}=\left\|R_{\mathrm{B}} R_{\mathrm{B}} \mathrm{B} f\right\|_{p} \leq C\|\mathrm{~B} f\|_{p} .
$$

By using the Fourier Bessel transformations, we have

$$
P\left(|y|^{2}\right) F_{\mathrm{B}}[(\mathrm{B}) f](y)=-|y|^{2} F_{\mathrm{B}}[P(\mathrm{~B}) f](y)
$$

(see [9]).

Corollary 3.5 Suppose $P_{k}$ is a homogeneous elliptic polynomial of degree $k$ and $f$ is $k$-times continuously differentiable with compact support. Then we have the priori estimate

$$
\|\mathrm{B} f\|_{p, \gamma} \leq A_{p}\left\|P_{k}(\mathrm{~B}) f\right\|_{p, \gamma}, \quad 1<p<\infty .
$$

To prove this inequality, we note that the following relation between Fourier Bessel transform of $\mathrm{B} f$ and $P_{k}(\mathrm{~B}) f$ holds:

$$
P_{k}\left(|y|^{2}\right) F_{\mathrm{B}}[\mathrm{B} f](y)=-|y|^{2} F_{\mathrm{B}}\left[P_{k}(\mathrm{~B}) f\right](y) .
$$

$P_{k}\left(|y|^{2}\right)$ is non-vanishing except at the origin and let $-\frac{|y|^{2}}{P_{k}\left(|y|^{2}\right)}$ be homogeneous of degree zero and indefinitely differentiable on the unit sphere. Then we get

$$
\mathrm{B} f=R_{\mathrm{B}}\left(P_{k}(\mathrm{~B}) f\right) .
$$

We also have the following $L_{p, \gamma}$ boundedness of the high order Riesz-Bessel transform.

Theorem 3.6 The high order Riesz-Bessel transforms generated by a Bessel-generalized shift operator are bounded operators from $L_{p, \gamma}\left(\mathbb{R}_{+}^{n}\right)$ into itself for all $1<p<\infty$

$$
\left\|R_{\mathrm{B}} f\right\|_{p, \gamma} \leq A_{p}\|f\|_{p, \gamma} .
$$

\section{Competing interests}

The authors declare that they have no competing interests.

Authors' contributions

All authors contributed equally to the writing of this paper. All authors read and approved the final manuscript.

\section{Author details}

'Department of Mathematics, Dumlupınar University, Kütahya, Turkey. ${ }^{2}$ Department of Electric-Electronic Engineering,

Faculty of Technology, Gazi University, Ankara, Turkey.

Received: 5 December 2013 Accepted: 27 March 2014 Published: 11 Apr 2014

\section{References}

1. Stein, EM, Weiss, G: Introduction to Fourier Analysis on Euclidean Spaces. Princeton University Press, Princeton (1971)

2. Muckenhoupt, B, Stein, EM: Classical expansions and their relation to conjugate harmonic functions. Trans. Am. Math. Soc. 118, 17-92 (1965)

3. Kipriyanov, IA: Fourier Bessel transformations and imbedding theorems. Trudy Math. Inst. Steklov 89, 130-213 (1967) (in Russian)

4. Kipriyanov, IA, Klyuchantsev, Ml: On singular integrals generated by the generalized shift operator II. Sib. Mat. Zh. 11, 1060-1083 (1970) (in Russian) 
5. Aliev, IA, Gadjiev, AD: Weighted estimates for multidimensional singular integrals generated by the generalized shift operator. Mat. Sb. 77(1), 37-55 (1994)

6. Guliev, VS: Sobolev theorems for B-Riesz potentials. Dokl. Akad. Nauk, Ross. Akad. Nauk 358(4), 450-451 (1998) (in Russian)

7. Aliev, IA: On Riesz transformations generated by generalized shift operator. Izv. Akad. Nauk Azerb. SSR, Ser. Fiz.-Teh. Mat. Nauk 8(1), 7-13 (1987)

8. Ekincioglu, I, Yıldırım, H, Akın, O: The mean value theorem for Laplacean-Bessel equation. In: Invited Lecture Delivered at the Seventh International Colloquium on Differential Equations, vol. II, pp. 29-37. Academic Publications, Plovdiv (1996)

9. Ekincioglu, I, Ozkın, IK: On higher order Riesz transformations generated by generalized shift operator. Turk. J. Math. 21, 51-60 (1997)

10. Gadjiev, AD, Guliev, EV: Two weighted inequality for singular integrals in Lebesgue spaces associated with the Laplace-Bessel differential operator. Proc. A. Razmadze Math. Inst. 138, 1-15 (2005)

11. Levitan, BM: Bessel function expansions in series and Fourier integrals. Usp. Mat. Nauk, 6(2), 102-143 (1951) (in Russian)

12. Kipriyanov, IA: Singular Elliptic Boundary Value Problems. Nauka, Moscow (1997) (in Russian)

13. Kipriyanov, IA: Boundary value problems for elliptic partial differential operators. Sov. Math. Dokl. 11, 1416-1419 (1970)

14. Cheng, M: On a theorem of Nicolesco and generalized Laplace operators. Proc. Am. Math. Soc. 2, 77-86 (1951)

15. Nicolesco, M: Les fonctions polyharmoniques. Actualitiés Sci. Ind. 4 (1936)

16. Pizetti, P: Sul significato geometrico del secondo parametro differenziale di una funzione sopra una superficie qualunque. Rend. Lincei 18, 309-316 (1909)

17. Picone, M: Nuovi indirizzi di ricerca teoria e nel calcolo soluzioni di talune equazioni lineari alle derivate parziali della fisica-matematica. Ann. Sc. Norm. Super. Pisa, Cl. Sci. 4, 213-288 (1936)

18. Kipriyanova, NI: Mean value theorems for polyharmonic equations. Non-classic problems. Math. Phys. Eq. NovoSibirsk, 81-85 (1984) (in Russian)

19. Kipriyanova, NI: The Mean Value Formula for Singular Differential Operator With Order Second. Differential Equations $121,11(1985)$

20. Betancor, JJ, Castro, AJ, Curbelo, J: Harmonic analysis operators associated with multidimensional Bessel operators. Proc. R. Soc. Edinb., Sect. A 142(5), 945-974 (2012)

21. Levitan, BM: The Theory of Generalized Translation Operators. Nauka, Moscow (1973) (in Russian)

22. Guliev, VS: Sobolev's theorem for the anisotropic Riesz-Bessel potential in Morrey-Bessel spaces. Dokl. Akad. Nauk, Ross. Akad. Nauk 367(2), 155-156 (1999) (in Russian)

23. Guliev, VS: Some properties of the anisotropic Riesz-Bessel potential. Anal. Math. 26(2), 99-118 (2000)

24. Guliev, VS, Garakhanova, NN, Zeren, Y: Pointwise and integral estimates for the Riesz B-potential in terms of B-maximal and B-fractionally maximal functions. Sib. Mat. Zh. 49(6), 1263-1279 (2008) (in Russian). Translation in Sib. Math. J. 49(6), 1008-1022 (2008)

25. Guliev, VS, Garakhanova, NN: The Sobolev-llin theorem for the Riesz B-potential. Sib. Mat. Zh. 50(1), 63-74 (2009) (in Russian). Translation in Sib. Math. J. 50(1), 49-59 (2009)

26. Kipriyanov, IA, Lyakhov, LN: Multipliers of the mixed Fourier-Bessel transform. Dokl. Akad. Nauk, Ross. Akad. Nauk 354(4), 449-451 (1997) (in Russian)

27. Lyakhov, LN: Multipliers of the mixed conversion of Fourier-Bessel. Proc. Steklov Inst. Math. 214, 234-249 (1997) (in Russian)

28. Lyakhov, LN, Raykhelgauz, LB: Singular heat equation with $D_{\mathrm{B} \gamma}$-Bessel operator. Fundamental solutions. Problems in mathematical analysis. No. 67. J. Math. Sci. (N.Y.) 188(3), 285-292 (2013)

29. Stempak, K, Torrera, JL: Higher Riesz transforms and imaginary powers associated to the harmonic oscillator. Acta Math. Hung. 111, 43-64 (2006)

30. Stein, EM: Singular Integrals and Differentiability Properties of Functions. Princeton University Press, Princeton (1970)

10.1186/1029-242X-2014-148

Cite this article as: Ekincioglu et al.: High order Riesz transforms and mean value formula for generalized translate operator. Journal of Inequalities and Applications 2014, 2014:148

\section{Submit your manuscript to a SpringerOpen ${ }^{\circ}$ journal and benefit from:}

- Convenient online submission

Rigorous peer review

- Immediate publication on acceptance

- Open access: articles freely available online

- High visibility within the field

- Retaining the copyright to your article 